\title{
Convergence of a Singular Euler-Maxwell Approximation of the Incompressible Euler Equations
}

\section{Jianwei Yang and Hongli Wang}

College of Mathematics and Information Science,

North China University of Water Resources and Electric Power, Zhengzhou 450011, China

Correspondence should be addressed to Jianwei Yang, yangjianwei@ncwu.edu.cn

Received 15 June 2011; Accepted 27 October 2011

Academic Editor: Vu Phat

Copyright (c) $2011 \mathrm{~J}$. Yang and H. Wang. This is an open access article distributed under the Creative Commons Attribution License, which permits unrestricted use, distribution, and reproduction in any medium, provided the original work is properly cited.

This paper studies the Euler-Maxwell system which is a model of a collisionless plasma. By energy estimation and the curl-div decomposition of the gradient, we rigorously justify a singular approximation of the incompressible Euler equations via a quasi-neutral regime.

\section{Introduction}

We study a model of a collisionless plasma where the ions are supposed to be at rest and create a neutralizing background field. The dynamics of the compressible electrons for plasma physics in a uniform background of nonmoving ions with fixed unit density obey the (scaled) one-fluid Euler-Maxwell system which takes the form [1]

$$
\begin{gathered}
\partial_{t} n+\operatorname{div}(n u)=0, \\
\partial_{t} u+(u \cdot \nabla) u=-(E+\gamma u \times B), \\
\gamma \lambda^{2} \partial_{t} E-\nabla \times B=\gamma n u, \quad \gamma \partial_{t} B+\nabla \times E=0, \\
\lambda^{2} \operatorname{div} E=1-n, \quad \operatorname{div} B=0,
\end{gathered}
$$

for $(x, t) \in \tau^{3} \times[0, T]$, subject to initial conditions:

$$
(n, u, E, B)(x, 0)=\left(n_{0}, u_{0}, E_{0}, B_{0}\right)(x)
$$


Here, $n, u$ denote the scaled macroscopic density and mean velocity vector of the electrons and $E, B$ the scaled electric field and magnetic field. They are functions of a three-dimensional position vector $x \in \tau^{3}$ and of the time $t>0$, where $\tau^{3}=(\mathbb{R} / 2 \pi \mathbb{Z})^{3}$ is the 3-dimensional torus. The fields $E$ and $B$ are coupled to the particles through the Maxwell equations and act on the particles via the Lorentz force $E+\gamma u \times B$. In the system (1.1)-(1.4), $j=n u$ stand for the current densities for the particle. Equations (1.1)-(1.2) are the mass and momentum balance laws, respectively, while (1.3)-(1.4) are the Maxwell equations.

The dimensionless parameters $\lambda>0$ and $\gamma>0$ can be chosen independently on each other, according to the desired scaling. Physically, $\lambda$ stands for the scaled Debye length, $\gamma$ can be chosen to be proportional $1 / c$, where $c$ is the speed of light. These parameters are small compared to the characteristic length of physical interest. Thus, regarding $\lambda$ and $\gamma$ as singular perturbation parameters, we can study the limits problem in the system (1.1)-(1.4) as these parameters tend to zero. Thus, the limit $\lambda \rightarrow 0$ is called the quasineutral limit while the limit $r \rightarrow 0$ is called the nonrelativistic limit.

Note that the systems are pressureless, and the only force is due to Lorentz force $E+$ $\gamma u \times B$. The energy of systems (1.1)-(1.4) is given by

$$
\boldsymbol{\varepsilon}^{\lambda}=\frac{1}{2} \int_{\tau^{3}}\left(n|u|^{2}+\lambda^{2}|E|^{2}+|B|^{2}\right) d x .
$$

In the present paper, we will consider the combined quasineutral and nonrelativistic limit of Euler-Maxwell system in the following scaling case:

$$
\gamma=\lambda^{2} \longrightarrow 0
$$

Passing to the limits when $\lambda \rightarrow 0$ and $\gamma \rightarrow 0$ go to zero, it is easy to obtain, at least at a very formal level, the incompressible Euler equations of ideal fluids:

$$
\begin{gathered}
\operatorname{div} u^{0}=0, \quad x \in \tau^{3}, t>0, \\
\partial_{t} u^{0}+u^{0} \cdot \nabla u^{0}+\nabla \phi^{0}=0, \quad x \in \tau^{3}, t>0, \\
u^{0}(t=0)=u_{0}^{0}, \quad x \in \tau^{3} .
\end{gathered}
$$

In these limits the plasma is expected to behave like an incompressible fluid, therefore governed by the incompressible Euler equation. The main aim of this note is to give a rigorous justification to this formal computation in the present paper.

Note that the above limit had been proven in [2] by an analysis of asymptotic expansions and a careful use of Modulated energy technique. In this paper, the case that the plasma is collisionless is considered. By energy estimation and the curl-div decomposition of the gradient, we obtain a convergence to the incompressible Euler system in $H^{s}$ norm for any $s$ large enough under the assumption that the initial data are well prepared.

There have been a lot of interesting results about the topic on the quasineutral limit, for the readers to see [3-8] for isentropic Euler-Poisson equations and $[9,10]$ for nonisentropic Euler-Poisson equations and therein references. For example, by using the method of formal asymptotic expansions, Peng et al. in [9] studied the quasineutral limit for Cauchy problems of multidimensional nonisentropic Euler-Poisson equations for plasmas or semiconductors 
with prepared initial data. Li in [10] justified the convergence of the nonisentropic EulerPoisson equation to the incompressible nonisentropic Euler type equation via the quasineutral limit and proved the local existence of smooth solutions to the limit equations by an iterative scheme. The Euler-Maxwell equations are more intricate than the Euler-Poisson equations for the complicated coupling of the Lorentz force. So there have been less studies on the Euler-Maxwell equations than the study on the Euler-Poisson equations. See [3, 6$8,10-16]$ and the references therein. The first rigorously study of the Euler-Maxwell equations with extrarelaxation terms is due to Chen et al. [17], where a global existence result to weak solutions in one-dimensional case is established by the fractional step Godunov scheme together with a compensated compactness argument. Jerome [18] establishes a local smooth solution theory for the Cauchy problem of compressible Hydrodynamic-Maxwell systems via a modification of the classical semigroup-resolvent approach of Kato. Paper [19] has just been studied for the convergence of one-fluid isentropic Euler-Maxwell system to compressible Euler-Poisson system via the nonrelativistic limit.

We split the rest of the paper in two sections: the first give the main result of this paper, and the second is devoted to justify the convergence of Euler-Maxwell equations to incompressible Euler equations by using the the curl-div decomposition of the gradient and the $\lambda$-weighted energy method.

\subsection{Notations and Preliminary Results}

Now we introduce some important notations and preliminary results that we will use in this paper as follows.

(1) Throughout this paper, $\nabla=\nabla_{x}$ is the gradient, $\alpha=\left(\alpha_{1}, \ldots, \alpha_{d}\right)$ and $\beta$, and so forth are multi-index, and $H^{s}\left(\tau^{3}\right)$ denotes the standard Sobolev space in torus $\tau^{3}$, which is defined by Fourier transform, namely, $f \in H^{s}\left(\tau^{3}\right)$ if and only if

$$
\|f\|_{s}^{2}=(2 \pi)^{d} \sum_{k \in \mathfrak{Z}^{d}}\left(1+|k|^{2}\right)^{s}|(\mp f)(k)|^{2}<+\infty,
$$

where $(\mathcal{F} f)(k)=\int_{\tau^{3}} f(x) e^{-i k x} d x$ is the Fourier transform of $f \in H^{s}\left(\tau^{3}\right)$. Noting that if $\int_{\tau^{3}} f(x) d x=0$, then $\|f\|_{L^{2}\left(\tau^{3}\right)} \leq\|\nabla f\|_{L^{2}\left(\tau^{3}\right)}$.

(2) The following vector analysis formulas will be repeatedly used, see [1]:

$$
\begin{gathered}
\operatorname{div}(f \times g)=\nabla \times f \cdot g-\nabla \times g \cdot f, \\
f \cdot \nabla g=(\nabla \times g) \times f+\nabla(f \cdot g)-\nabla f \cdot g, \quad \nabla f \cdot g=\sum_{j=1}^{3} \nabla f_{j} g_{j}, \\
f \cdot \nabla f=(\nabla \times f) \times f+\nabla\left(\frac{|f|^{2}}{2}\right), \\
\nabla \times(f \times g)=f \operatorname{div} g-g \operatorname{div} f+(g \cdot \nabla) f-(f \cdot \nabla) g .
\end{gathered}
$$




\section{The Main Result}

First, we recall the classical result on the existence of sufficiently regular solutions of the incompressible Euler equations (see [20]).

Proposition 2.1. Let $u_{0}^{0}$ be a divergence-free vector field on $\tau^{3}$ and satisfy $u_{0}^{0} \in H^{s+2}, s>3 / 2+2$. Then there exist $0<T \leq \infty$, the maximal existence time, and a unique smooth solution $\left(u^{0}, \phi^{0}\right)$ of the incompressible Euler equation (1.8)-(1.9) on [0,T) satisfying that $\int_{\tau^{3}} \phi^{0}(t, x) d x=0$, and, for any $T_{0}<T$, the inequality

$$
\sup _{0 \leq t \leq T_{0}}\left(\left\|u^{0}\right\|_{H^{s+2}}+\left\|\partial_{t} u^{0}\right\|_{H^{s+1}}+\left\|\nabla \phi^{0}\right\|_{H^{s+2}}+\left\|\partial_{t} \nabla \phi^{0}\right\|_{H^{s+1}}\right) \leq C\left(T_{0}\right)
$$

holds for some positive constant $C\left(T_{0}\right)$.

We will prove the following result.

Theorem 2.2. Let $s \geq 3 / 2+2$ with $s \in \mathbb{N}$. Assume that $n_{0}=1, u_{0}=u_{0}^{0}+\lambda u_{0}^{1}$ with $\operatorname{div} u_{0}^{0}=0, u_{0}^{0} \in$ $H^{s+2}, u_{0}^{1} \in H^{s}, s>3 / 2+2,\left\|u_{0}^{1}\right\|_{s} \leq M_{1}$ uniformly in $\lambda$. Let $T$ be the maximal existence time of smooth solution $\left(u^{0}, \phi^{0}\right)$ of the incompressible Euler equation (1.8)-(1.10). Then for any $T_{0}<T$, there exist constants $\lambda_{0}\left(T_{0}\right)$ and $M\left(T_{0}\right)$, depending only on $T_{0}$ and the initial data, such that Euler-Maxwell system (1.1)-(1.5) has a classical smooth solution $(n, u, E, B)$, defined on $\left[0, T_{0}\right]$, satisfying

$$
\left\|\lambda^{-2}(n-1)(t, \cdot)\right\|_{s-1}+\left\|\lambda^{-1}\left(u-u^{0}\right)(t, \cdot)\right\|_{s}+\lambda^{2}\left\|\left(E-\nabla \phi^{0}\right)(t, \cdot)\right\|_{s}+\lambda\|B(t, \cdot)\|_{s} \leq M\left(T_{0}\right),
$$

for all $0<\lambda \leq \lambda_{0}$ and $0 \leq t \leq T_{0}$.

\section{The Proof of Theorem 2.2}

Set $(n, u, E, B)$ to be the classical solutions to the problem (1.1)-(1.5), and set

$$
(n, u, E, B)=\left(1+\lambda^{2} n^{1}-\lambda^{2} \Delta \phi^{0}, u^{0}+\lambda u^{1}, \nabla \phi^{0}+E^{1}, B^{1}\right) .
$$

Obviously the vector $\left(n^{1}, u^{1}, E^{1}, B^{1}\right)$ solves the error system

$$
\begin{gathered}
\partial_{t} n^{1}+u \cdot \nabla n^{1}+\frac{\operatorname{div} u^{1}}{\lambda}+\lambda\left(n^{1}-\Delta \phi^{0}\right) \operatorname{div} u^{1}=\left(\partial_{t}+u \cdot \nabla\right) \Delta \phi^{0}, \\
\partial_{t} u^{1}+(u \cdot \nabla) u^{1}=-\left(u^{1} \cdot \nabla\right) u^{0}-\frac{E^{1}}{\lambda}-\lambda u \times B^{1}, \\
\lambda^{4} \partial_{t} E^{1}-\nabla \times B^{1}=\lambda^{2} n u-\lambda^{4} \partial_{t} \nabla \phi^{0}, \quad \operatorname{div} E^{1}=-n^{1}, \\
\lambda^{2} \partial_{t} B^{1}+\nabla \times E^{1}=0, \quad \operatorname{div} B^{1}=0, \\
\left.\left(n^{1}, u^{1}, E^{1}, B^{1}\right)\right|_{t=0}=\left(n_{0}^{1}, u_{0}^{1}, E_{0}^{1}, B_{0}^{1}\right)(x),
\end{gathered}
$$


for $x \in \tau^{3}, t>0$ and with the initial comparable condition

$$
\operatorname{div} E_{0}^{1}=-n_{0}^{1}, \quad \operatorname{div} B_{0}^{1}=0 .
$$

\subsection{Reformulation of the System (3.2) with New Unknowns}

Now we make the following change of unknowns:

$$
\left(d^{1}, \omega^{1}\right)=\left(\operatorname{div} u^{1}, \nabla \times u^{1}\right),
$$

then one gets

$$
\operatorname{div} u=\lambda d^{1}, \quad \nabla \times u=\omega=\omega^{0}+\lambda \omega^{1},
$$

with $\omega^{0}=\nabla \times u^{0}$.

Taking the divergence of the second equation in (3.2) and noting that $\operatorname{div} E^{1}=-n^{1}$, one gets

$$
\partial_{t} d^{1}+u \cdot \nabla d^{1}-\frac{n^{1}}{\lambda}=-2 D u^{0}: D u^{1}+\lambda D u^{1}: D u^{1}-\lambda \operatorname{div}\left(u \times B^{1}\right)
$$

Taking the curl of the second equation in (3.2), with the aid of the vector analysis formulas given in Section 1, by a direction calculation, we have

$$
\partial_{t}\left(\nabla \times u^{1}\right)+\nabla \times\left((u \cdot \nabla) u^{1}\right)+\nabla \times\left(\left(u^{1} \cdot \nabla\right) u^{0}\right)=\lambda^{2} \partial_{t} B^{1}-\lambda \nabla \times\left(u \times B^{1}\right) .
$$

Here we have used the error equation $\lambda^{2} \partial_{t} B^{1}+\nabla \times E^{1}=0$ of the magnetic field in the error system (3.2). Since $\operatorname{div} B^{1}=0$, there exists a vector function $b^{1}$ such that

$$
B^{1}=\nabla \times b^{1}
$$

Then, we have

$$
\partial_{t}\left(\nabla \times\left(u^{1}-\lambda b^{1}\right)\right)+\nabla \times\left(\left(\nabla \times u^{1}-\lambda b^{1}\right) \times u\right)=\nabla \times\left(\nabla u^{0} \cdot u^{1}\right)-\nabla \times\left(u^{1} \cdot \nabla u^{0}\right) .
$$

Next introduce the general vorticity

$$
\Omega^{1}=\omega^{1}-\lambda \nabla \times b^{1}=\nabla \times\left(u^{1}-\lambda b^{1}\right) .
$$

By using the vector analysis formulas (1.15), then it follows from the above that $\Omega^{1}$ satisfies the following vorticity equation:

$$
\partial_{t} \Omega^{1}+(u \cdot \nabla) \Omega^{1}-\left(\Omega^{1} \cdot \nabla\right) u-\lambda d^{1} \Omega^{1}-\nabla \times\left(\nabla u^{0} \cdot u^{1}\right)+\nabla \times\left(\nabla u^{1} \cdot u^{0}\right)=0 .
$$


Therefore, we can rewrite the error system (3.2) as follows:

$$
\begin{gathered}
\partial_{t} n^{1}+u \cdot \nabla n^{1}+\frac{d^{1}}{\lambda}=\left(\partial_{t}+u \cdot \nabla\right) \Delta \phi^{0}-\lambda\left(n^{1}-\Delta \phi^{0}\right) d^{1}, \\
\partial_{t} d^{1}+u \cdot \nabla d^{1}-\frac{n^{1}}{\lambda}=-2 D u^{0}: D u^{1}-\lambda D u^{1}: D u^{1}-\lambda \operatorname{div}\left(u \times B^{1}\right), \\
\partial_{t} \Omega^{1}+(u \cdot \nabla) \Omega^{1}-\left(\Omega^{1} \cdot \nabla\right) u+\lambda d^{1} \Omega^{1}=\nabla \times\left(\nabla u^{0} \cdot u^{1}\right)-\nabla \times\left(u^{1} \cdot \nabla u^{0}\right), \\
\lambda^{4} \partial_{t} E^{1}-\nabla \times B^{1}=\lambda^{2} n u, \quad \operatorname{div} E^{1}=-n^{1}, \\
\lambda^{2} \partial_{t} B^{1}+\nabla \times E^{1}=0, \quad \operatorname{div} B^{1}=0, \\
\left.\left(n^{1}, u^{1}, E^{1}, B^{1}\right)\right|_{t=0}=\left(n_{0}^{1}, u_{0}^{1}, E_{0}^{1}, B_{0}^{1}\right)(x) .
\end{gathered}
$$

In order to prove the Theorem 2.2, we introduce the Sobolev norms:

$$
\begin{gathered}
\|\cdot\|=\|\cdot\|_{L^{2}\left(\tau^{3}\right)}, \\
\left\|W^{\lambda}(t)\right\|_{s}=\left(\left\|n^{1}(t)\right\|_{s-1}^{2}+\left\|u^{1}(t)\right\|_{s}^{2}+\lambda^{2}\left\|E^{1}(t)\right\|_{s}^{2}+\lambda\left\|B^{1}(t)\right\|_{s}^{2}\right)^{1 / 2} .
\end{gathered}
$$

Next, one begins to estimate $\left\|W^{\lambda}\right\|_{s}$. Our basic idea is to overcome the singularity which is caused by $d^{1} / \lambda$ and $n^{1} / \lambda$ by using the special structures between the first equation and the the second equation in the system (3.6).

\subsection{Energy Estimates}

Now we control $\left(\left\|n^{1}(t)\right\|_{s-1}^{2}+\left\|d^{1}(t)\right\|_{s-1}^{2}+\left\|\omega^{1}(t)\right\|_{s-1}^{2}\right)$.

Lemma 3.1. Let $s$ be an integer with $s>3 / 2+2$. Then for any $0<t<T$ with $T \in\left(0, T_{0}\right]$, one has

$$
\begin{aligned}
\left(\left\|n^{1}(t)\right\|_{s-1}^{2}+\left\|d^{1}(t)\right\|_{s-1}^{2}+\left\|\omega^{1}(t)\right\|_{s-1}^{2}\right) & \\
\leq & C\left(\left\|n^{1}(t=0)\right\|_{s-1}^{2}+\left\|d^{1}(t=0)\right\|_{s-1}^{2}+\left\|\omega^{1}(t=0)\right\|_{s-1}^{2}\right) \\
& +C \int_{0}^{t}\left(1+\left\|W^{\lambda}(\tau)\right\|_{s}^{2}+\left\|W^{\lambda}(\tau)\right\|_{s}^{3}\right) d \tau .
\end{aligned}
$$

Proof. Let $\alpha \in \mathbb{N}^{3}$ with $|\alpha| \leq s-1$ and $s>3 / 2+2$. Taking $\partial_{x}^{\alpha}$ of (3.12) and multiplying the resulting equation by $\partial_{x}^{\alpha} n^{1}$, by integration by parts, we have

$$
\begin{aligned}
\frac{d}{d t}\left\|\partial_{x}^{\alpha} n^{1}\right\|^{2}= & \left(\operatorname{div} u \partial_{x}^{\alpha} n^{1}, \partial_{x}^{\alpha} n^{1}\right)+2\left(\mathcal{L}_{\alpha}^{(1)}, \partial_{x}^{\alpha} n^{1}\right)-\frac{2}{\lambda}\left(\partial_{x}^{\alpha} d^{1}, \partial_{x}^{\alpha} n^{1}\right) \\
& +2\left(\partial_{x}^{\alpha}\left(\left(\partial_{t}+u \cdot \nabla\right) \Delta \phi^{0}-\lambda\left(n^{1}-\Delta \phi^{0}\right) d^{1}\right), \partial_{x}^{\alpha} n^{1}\right)
\end{aligned}
$$


Journal of Applied Mathematics

where the commutator

$$
\mathscr{H}_{\alpha}^{(1)}=-\left[\partial_{x}^{\alpha}\left((u \cdot \nabla) n^{1}\right)-u \cdot \partial_{x}^{\alpha} \nabla n^{1}\right]
$$

which can be estimated as follows:

$$
\begin{aligned}
\left\|\mathscr{L}_{\alpha}^{(1)}\right\| & =C\|\nabla u\|_{L^{\infty}}\left\|\partial_{x}^{s-2} \nabla n^{1}\right\|+C\left\|\nabla n^{1}\right\|_{L^{\infty}}\left\|\partial_{x}^{s-1} u\right\| \\
& \leq C\|\nabla u\|_{s^{-1}}\left\|\partial_{x}^{s-2} \nabla n^{1}\right\|+C\left\|\nabla n^{1}\right\|_{s-2}\left\|\partial_{x}^{s-1} u\right\| \\
& \leq C\left(1+\lambda\left\|W^{\lambda}(t)\right\|_{s}\right)\left\|W^{\curlywedge}(t)\right\|_{s} .
\end{aligned}
$$

Here we used the basic Moser-type calculus inequalities [21, 22]:

$$
\left\|D_{x}^{\alpha}(f g)-f D_{x}^{\alpha} g\right\|_{L^{2}} \leq C_{s}\left(\left\|D_{x} f\right\|_{L^{\infty}}\left\|D_{x}^{s-1} g\right\|_{L^{2}}+\|g\|_{L^{\infty}}\left\|D_{x}^{s} f\right\|_{L^{2}}\right), \quad s \geq 1 .
$$

Hence, by Cauchy-Schwartz's inequality and the Sobolev lemma, noting $\operatorname{div} u=\lambda d^{1}$, one gets

$$
\begin{aligned}
& \left(\operatorname{div} u \partial_{x}^{\alpha} n^{1}, \partial_{x}^{\alpha} n^{1}\right)+2\left(\mathscr{H}_{\alpha}^{(1)}, \partial_{x}^{\alpha} n^{\lambda}\right) \leq C\left(1+\left\|W^{\lambda}(t)\right\|_{s}^{2}+\lambda\left\|W^{\lambda}(t)\right\|_{s}^{3}\right), \\
& 2\left(\partial_{x}^{\alpha}\left(\left(\partial_{t}+u \cdot \nabla\right) \Delta \phi^{0}-\lambda\left(n^{1}-\Delta \phi^{0}\right) d^{1}\right), \partial_{x}^{\alpha} n^{1}\right) \leq C\left(1+\left\|W^{\lambda}(t)\right\|_{s}^{2}\right) .
\end{aligned}
$$

Combining (3.20) with (3.24), one obtains

$$
\frac{d}{d t}\left\|\partial_{x}^{\alpha} n^{1}\right\|^{2} \leq C\left(1+\left\|W^{\lambda}(t)\right\|_{s}^{2}+\lambda\left\|W^{\lambda}(t)\right\|_{s}^{3}\right)-\frac{2}{\lambda}\left(\partial_{x}^{\alpha} d^{1}, \partial_{x}^{\alpha} n^{1}\right)
$$

Next, Taking $\partial_{x}^{\alpha}$ of (3.13) and multiplying the resulting equation by $\partial_{x}^{\alpha} d^{1}$, by integration by parts, we have

$$
\begin{aligned}
\frac{d}{d t}\left\|\partial_{x}^{\alpha} d^{1}\right\|^{2}= & \left(\operatorname{div} u \partial_{x}^{\alpha} d^{1}, \partial_{x}^{\alpha} d^{1}\right)+2\left(\mathscr{\ell}_{\alpha}^{(2)}, \partial_{x}^{\alpha} d^{1}\right)+\frac{2}{\lambda}\left(\partial_{x}^{\alpha} d^{1}, \partial_{x}^{\alpha} n^{1}\right) \\
& -2\left(\partial_{x}^{\alpha}\left(2 D u^{0}: D u^{1}+\lambda D u^{1}: D u^{1}\right), \partial_{x}^{\alpha} d^{1}\right)+\lambda\left(\partial_{x}^{\alpha}\left(\nabla \times\left(u \times B^{1}\right)\right), \partial_{x}^{\alpha} d^{1}\right),
\end{aligned}
$$

where the commutator

$$
\mathscr{\ell}_{\alpha}^{(2)}=-\left[\partial_{x}^{\alpha}\left((u \cdot \nabla) u^{1}\right)-u \cdot \partial_{x}^{\alpha} \nabla u^{1}\right]
$$


which can be estimated by

$$
\begin{aligned}
\left\|\mathscr{\ell}_{\alpha}^{(2)}\right\| & =C\|\nabla u\|_{L^{\infty}}\left\|\partial_{x}^{s-2} \nabla u^{1}\right\|+C\left\|\nabla u^{1}\right\|_{L^{\infty}}\left\|\partial_{x}^{s-1} u\right\| \\
& \leq C\|\nabla u\|_{s^{-1}}\left\|\partial_{x}^{s-2} \nabla u^{1}\right\|+C\left\|\nabla u^{1}\right\|_{s-2}\left\|\partial_{x}^{s-1} u\right\| \\
& \leq C\left(1+\lambda\left\|W^{\lambda}(t)\right\|_{s}\right)\left\|W^{\lambda}(t)\right\|_{s}
\end{aligned}
$$

For the first, forth, and fifth terms, using the property of the approximate solution of incompressible Euler equations (1.8)-(1.10), Cauchy-Schwarz's inequality, and the Sobolev lemma, we get

$$
\begin{gathered}
\left(\operatorname{div} u \partial_{x}^{\alpha} d^{1}, \partial_{x}^{\alpha} d^{1}\right) \leq C\left(1+\lambda\left\|W^{\lambda}(t)\right\|_{s}\right)\left\|W^{\lambda}(t)\right\|_{s^{\prime}}^{2} \\
-2\left(\partial_{x}^{\alpha}\left(2 D u^{0}: D u^{1}+\lambda D u^{1}: D u^{1}\right), \partial_{x}^{\alpha} d^{1}\right) \leq C\left(1+\lambda\left\|W^{\lambda}(t)\right\|_{s}\right)\left\|W^{\lambda}(t)\right\|_{s^{\prime}}^{2} \\
\lambda\left(\partial_{x}^{\alpha}\left(\nabla \times\left(u \times B^{1}\right)\right), \partial_{x}^{\alpha} d^{1}\right) \leq C\left(1+\lambda\left\|W^{\lambda}(t)\right\|_{s}\right)\left\|W^{\lambda}(t)\right\|_{s}^{2} .
\end{gathered}
$$

For the second term, using the estimate (3.28) for $\mathfrak{H}_{\alpha}^{(2)}$, we obtain

$$
2\left(\mathscr{H}_{\alpha}^{(2)}, \partial_{x}^{\alpha} d^{1}\right) \leq C\left(1+\lambda\left\|W^{\lambda}(t)\right\|_{s}\right)\left\|W^{\lambda}(t)\right\|_{s}^{2}
$$

Combining (3.26) with (3.29)-(3.30), we have

$$
\frac{d}{d t}\left\|\partial_{x}^{\alpha} d^{1}\right\|^{2} \leq C\left(1+\lambda\left\|W^{\lambda}(t)\right\|_{s}\right)\left\|W^{\lambda}(t)\right\|_{s}^{2}+\frac{2}{\lambda}\left(\partial_{x}^{\alpha} d^{1}, \partial_{x}^{\alpha} n^{1}\right) .
$$

From (3.25) and (3.31), we can get

$$
\frac{d}{d t}\left(\left\|\partial_{x}^{\alpha} n^{1}\right\|^{2}+\left\|\partial_{x}^{\alpha} d^{1}\right\|^{2}\right) \leq C\left(1+\left\|W^{\lambda}(t)\right\|_{s}^{2}+\lambda\left\|W^{\lambda}(t)\right\|_{s}^{3}\right)
$$

which leads to

$$
\begin{aligned}
\left(\left\|n^{1}(t)\right\|_{s-1}^{2}+\left\|d^{1}(t)\right\|_{s-1}^{2}\right) \leq & C\left(\left\|n^{1}(t=0)\right\|_{s-1}^{2}+\left\|d^{1}(t=0)\right\|_{s-1}^{2}\right) \\
& +C \int_{0}^{t}\left(1+\left\|W^{\lambda}(\tau)\right\|_{s}^{2}+\lambda\left\|W^{\lambda}(\tau)\right\|_{s}^{3}\right) d \tau .
\end{aligned}
$$


Taking Taking $\partial_{x}^{\alpha}$ on (3.14) and multiplying the resulting equation by $\partial_{x}^{\alpha}$, by integration by parts, we have

$$
\begin{aligned}
\frac{d}{d t}\left\|\partial_{x}^{\alpha} \Omega^{1}\right\|^{2}= & \left(\operatorname{div} u \partial_{x}^{\alpha} \Omega^{1}, \partial_{x}^{\alpha} \Omega^{1}\right)-2\left(\partial_{x}^{\alpha}\left(\left(\Omega^{1} \cdot \nabla\right) u-\lambda d^{1} \Omega^{1}\right), \partial_{x}^{\alpha} \Omega^{1}\right) \\
& +2\left(\partial_{x}^{\alpha}\left(\nabla \times\left(\nabla u^{0} \cdot u^{1}\right)-\nabla \times\left(u^{1} \cdot \nabla u^{0}\right)\right), \partial_{x}^{\alpha} \Omega^{1}\right)+2\left(\mathscr{l}_{\alpha}^{(3)}, \partial_{x}^{\alpha} \Omega^{1}\right),
\end{aligned}
$$

where the commutator

$$
\mathscr{H}_{\alpha}^{(3)}=-\left[\partial_{x}^{\alpha}\left((u \cdot \nabla) \Omega^{1}\right)-u \cdot \partial_{x}^{\alpha} \nabla \Omega^{1}\right]
$$

which can be estimated by

$$
\begin{aligned}
\left\|\mathscr{L}_{\alpha}^{(3)}\right\| & =C\|\nabla u\|_{L^{\infty}}\left\|\partial_{x}^{s-2} \nabla \Omega^{1}\right\|+C\left\|\nabla u^{1}\right\|_{L^{\infty}}\left\|\partial_{x}^{s-1} \Omega\right\| \\
& \leq C\|\nabla u\|_{s^{-1}}\left\|\partial_{x}^{s-2} \nabla \Omega^{1}\right\|+C\left\|\nabla \Omega^{1}\right\|_{s-2}\left\|\partial_{x}^{s-1} u\right\| \\
& \leq C\left(1+\lambda\left\|W^{\lambda}(t)\right\|_{s}\right)\left\|\Omega^{1}(t)\right\|_{s-1} .
\end{aligned}
$$

Using the estimates (3.36) for $\mathfrak{H}_{\alpha}^{(3)}$, we have, with the aid of Cauchy-Schwarz's inequality and the Sobolev lemma,

$$
\begin{gathered}
\left(\operatorname{div} u \partial_{x}^{\alpha} \Omega^{1}, \partial_{x}^{\alpha} \Omega^{1}\right) \leq C\left(1+\lambda\left\|W^{\lambda}(t)\right\|_{s}\right)\left\|\Omega^{1}(t)\right\|_{s-1^{\prime}}^{2} \\
-2\left(\partial_{x}^{\alpha}\left(\left(\Omega^{1} \cdot \nabla\right) u-\lambda d^{1} \Omega^{1}\right), \partial_{x}^{\alpha} \Omega^{1}\right) \leq C\left(1+\lambda\left\|W^{\lambda}(t)\right\|_{s}\right)\left\|\Omega^{1}(t)\right\|_{s-1^{\prime}}^{2} \\
2\left(\partial_{x}^{\alpha}\left(\nabla \times\left(\nabla u^{0} \cdot u^{1}\right)-\nabla \times\left(u^{1} \cdot \nabla u^{0}\right)\right), \partial_{x}^{\alpha} \Omega^{1}\right) \leq C\left(1+\left\|W^{\lambda}(t)\right\| \|_{s}\right)\left\|\Omega^{1}(t)\right\|_{s-1^{\prime}}^{2} \\
2\left(\mathcal{L}_{\alpha}^{(3)}, \partial_{x}^{\alpha} \Omega^{1}\right) \leq C\left(1+\lambda\left\|W^{\lambda}(t)\right\|_{s}\right)\left\|\Omega^{1}(t)\right\|_{s-1}^{2} .
\end{gathered}
$$

Combining (3.34) with (3.37), we get

$$
\frac{d}{d t}\left\|\partial_{x}^{\alpha} \Omega^{1}\right\|^{2} \leq C\left(1+\left\|W^{\lambda}(t)\right\|_{s}\right)\left\|\Omega^{1}(t)\right\|_{s-1^{\prime}}^{2}
$$

which yields, for any $0<t<T$,

$$
\left\|\Omega^{1}\right\|_{s-1}^{2} \leq\left\|\Omega^{1}(t=0)\right\|_{s-1}^{2}+C \int_{0}^{t}\left(1+\left\|W^{\lambda}(\tau)\right\|_{s}\right)\left\|\Omega^{1}(\tau)\right\|_{s-1}^{2} d \tau .
$$


Noting the definition of $\Omega^{1}$ (see (3.10)), we obtain

$$
\begin{aligned}
& \left\|\omega^{1}\right\|_{s-1}^{2} \leq 2\left\|\Omega^{1}\right\|_{s-1}^{2}+2 \lambda^{2}\left\|B^{1}\right\|_{s-1}^{2} \\
& \left\|\Omega^{1}\right\|_{s-1}^{2} \leq 2\left\|\omega^{1}\right\|_{s-1}^{2}+2 \lambda^{2}\left\|B^{1}\right\|_{s-1}^{2} .
\end{aligned}
$$

Then (3.39)-(3.40) give the estimate

$$
\begin{aligned}
\left\|\omega^{1}\right\|_{s-1}^{2} \leq & C\left(\left\|\Omega^{1}(t=0)\right\|_{s-1}^{2}+\lambda^{2}\left\|B^{1}(t=0)\right\|_{s-1}^{2}\right)+C \lambda^{2}\left\|B^{1}\right\|_{s-1}^{2} \\
& +C \int_{0}^{t}\left(\left(1+\left\|W^{\lambda}(\tau)\right\|_{s}\right)\left\|\omega^{1}(\tau)\right\|_{s-1}^{2}+\lambda^{2}\left\|B^{1}\right\|_{s-1}^{2}\right) d \tau .
\end{aligned}
$$

So, from (3.33) and (3.41) we complete the proof of Lemma 3.2. lows.

Next, we obtain the high order energy estimates on the electric-magnetic field as fol-

Lemma 3.2. Let $s$ be an integer with $s>3 / 2+2$. Then for any $0<t<T$ with $T \in\left(0, T_{0}\right]$, one has

$$
\begin{aligned}
\left(\lambda^{4}\left\|E^{1}(t)\right\|_{s}^{2}+\lambda^{2}\left\|B^{1}(t)\right\|_{s}^{2}\right) \leq & C\left(\lambda^{4}\left\|E^{1}(t=0)\right\|_{s}^{2}+\lambda^{2}\left\|B^{1}(t=0)\right\|_{s}^{2}\right) \\
& +C \int_{0}^{t}\left(1+\left\|W^{\lambda}(\tau)\right\|_{s}^{2}+\left\|W^{\lambda}(\tau)\right\|_{s}^{3}\right) d \tau .
\end{aligned}
$$

Proof. Let $\beta \in \mathbb{N}^{3}$ with $|\beta| \leq s$ and $s>3 / 2+2$. Taking $\partial_{x}^{\beta}$ of (3.15) and then taking the $L^{2}$ inner product of the resulting equation with $\partial_{x}^{\beta} E^{1}$, and taking $\partial_{x}^{\beta}$ of (3.16) and then taking the $L^{2}$ inner product of the resulting equation with $\partial_{x}^{\beta} B^{1}$, and summing them up, we get

$$
\begin{aligned}
& \frac{d}{d t}\left(\lambda^{4}\left\|\partial_{x}^{\beta} E^{1}\right\|^{2}+\lambda^{2}\left\|\partial_{x}^{\beta} B^{1}\right\|^{2}\right) \\
& \quad=2 \lambda^{2}\left(\partial_{x}^{\beta}\left(\left(1-\lambda^{2} \Delta \phi^{0}\right) u\right), \partial_{x}^{\beta} E^{1}\right)+2 \lambda^{4}\left(\partial_{x}^{\beta}\left(n^{1} u\right), \partial_{x}^{\beta} E^{1}\right) \\
& \leq C\left(1+\left\|W^{\lambda}(t)\right\|_{s}^{2}\right)-2 \lambda^{4}\left(u \partial_{x}^{\beta}\left(\operatorname{div} E^{1}\right), \partial_{x}^{\beta} E^{1}\right)-2 \lambda^{4}\left(\varkappa_{\beta}, \partial_{x}^{\beta} E^{1}\right),
\end{aligned}
$$

where the commutator is defined by

$$
\mathscr{\ell}_{\beta}=\partial_{x}^{\beta}\left(u \operatorname{div} E^{1}\right)-u \partial_{x}^{\beta}\left(\operatorname{div} E^{1}\right),
$$


Journal of Applied Mathematics

which can be estimated as follows

$$
\begin{aligned}
H_{\beta} & \leq C\left(\|\nabla u\|_{L^{\infty}}\left\|\partial_{x}^{s-1} \operatorname{div} E^{1}\right\|+\left\|\operatorname{div} E^{1}\right\|_{L^{\infty}}\left\|\partial_{x}^{s} u\right\|\right) \\
& \leq C\left(\|\nabla u\|_{s^{-1}}\left\|\partial_{x}^{s-1} \operatorname{div} E^{1}\right\|+\left\|\operatorname{div} E^{1}\right\|_{s-1}\left\|\partial_{x}^{s} u\right\|\right) \\
& =C\left(1+\lambda\left\|W^{\lambda}(t)\right\|_{s}\right)\left\|E^{1}\right\|_{s} .
\end{aligned}
$$

Here we have used Sobolev lemma, $s>3 / 2+2$ and the vector analysis formula (1.12).

With the aid of Hölder's inequality, we have, using the estimate (3.45) of the commutator $\mathscr{d}_{\beta}$, that

$$
-2 \lambda^{4}\left(\mathscr{L}_{\beta}, \partial_{x}^{\beta} E^{1}\right) \leq C\left(1+\lambda\left\|W^{\lambda}(t)\right\|_{s}\right)\left\|W^{\lambda}(t)\right\|_{s}^{2}
$$

By integration by parts and Sobolev lemma,

$$
-2 \lambda^{4}\left(u \partial_{x}^{\beta}\left(\operatorname{div} E^{1}\right), \partial_{x}^{\beta} E^{1}\right) \leq C\left(1+\lambda\left\|W^{\lambda}(t)\right\|_{s}\right)\left\|W^{\lambda}(t)\right\|_{s}^{2} .
$$

Combining (3.43) with (3.46) and (3.47), one gets

$$
\frac{d}{d t}\left(\lambda^{4}\left\|\partial_{x}^{\beta} E^{1}(t)\right\|^{2}+\lambda^{2}\left\|\partial_{x}^{\beta} B^{1}(t)\right\|^{2}\right) \leq C\left(1+\left\|W^{\lambda}(t)\right\|_{s}^{2}+\lambda\left\|W^{\lambda}(t)\right\|_{s}^{3}\right)
$$

which yields (3.42).

\subsection{The End of Proof of Theorem 2.2}

Now, we introduce an $\lambda$-weighted Sobolev type energy function

$$
\varepsilon^{\lambda}(t)=\left\|W^{\lambda}(t)\right\|_{s}^{2}
$$

Then it follows from the estimates (3.19), (3.42), and the curl-divergence decomposition inequality

$$
\left\|\nabla u^{1}\right\|_{s-1} \leq C\left(\left\|d^{1}\right\|_{s-1}+\left\|\Omega^{1}\right\|_{s-1}\right)
$$

that there exists an $\lambda_{0}>0$, depending only upon $T_{0}$, such that, for any $0<\lambda \leq \lambda_{0}$ and any $0<t<T$,

$$
\varepsilon^{\lambda}(t) \leq C \varepsilon^{\lambda}(t)(t=0)+C \int_{0}^{t}\left(1+\varepsilon^{\lambda}(\tau)+\left(\varepsilon^{\lambda}(\tau)\right)^{3 / 2}\right) d \tau
$$


Since $\varepsilon^{\lambda}(t=0) \leq M_{0}$ for some positive constant, now applying Gronwall's inequality to (3.51), one can conclude that there exists an $\lambda_{0}$ sufficiently small such that for any $\lambda \leq \lambda_{0}$ and $0<t<T$,

$$
\mathcal{E}^{\lambda}(t) \leq M_{0}
$$

which gives the desired priori estimate

$$
\|\| W^{\lambda}(t) \|\left.\right|_{s, T_{0}} \leq M\left(T_{0}\right)
$$

Finally, we can return to the problem (1.1)-(1.4) to conclude our Theorem 2.2. In fact, the assumptions in Theorem 2.2 imply that the estimate (3.53) holds. Thus, it follows from the estimate (3.53) and the transform (3.1) that the estimate (2.2) holds. The proof of Theorem 2.2 is complete.

\section{Acknowledgment}

The author is grateful for the support from the National Science Foundation of China (Grant no. 10771099) and the Beijing Science Foundation (Grant no. 1082001).

\section{References}

[1] F. Chen, Introduction to Plasma Physics and Controlled Fusion, vol. 1, Plenum Press, New York, NY, USA, 1984.

[2] Y. J. Peng and S. Wang, "Convergence of compressible Euler-Maxwell equations to incompressible Euler equations," Communications in Partial Differential Equations, vol. 33, no. 1-3, pp. 349-376, 2008.

[3] S. Cordier and E. Grenier, "Quasineutral limit of an Euler-Poisson system arising from plasma physics," Communications in Partial Differential Equations, vol. 25, no. 5-6, pp. 1099-1113, 2000.

[4] G. Loeper, "Quasi-neutral limit of the Euler-Poisson and Euler-Monge-Ampère systems," Communications in Partial Differential Equations, vol. 30, no. 7-9, pp. 1141-1167, 2005.

[5] E. Grenier, "Pseudo-differential energy estimates of singular perturbations," Communications on Pure and Applied Mathematics, vol. 50, no. 9, pp. 821-865, 1997.

[6] Y. J. Peng and Y. G. Wang, "Convergence of compressible Euler-Poisson equations to incompressible type Euler equations," Asymptotic Analysis, vol. 41, no. 2, pp. 141-160, 2005.

[7] M. Slemrod and N. Sternberg, "Quasi-neutral limit for Euler-Poisson system," Journal of Nonlinear Science, vol. 11, no. 3, pp. 193-209, 2001.

[8] S. Wang, "Quasineutral limit of Euler-Poisson system with and without viscosity," Communications in Partial Differential Equations, vol. 29, no. 3-4, pp. 419-456, 2004.

[9] Y. J. Peng, Y.-G. Wang, and W. A. Yong, "Quasi-neutral limit of the non-isentropic Euler-Poisson system," Proceedings of the Royal Society of Edinburgh, Section A, vol. 136, no. 5, pp. 1013-1026, 2006.

[10] Y. Li, "Convergence of the nonisentropic Euler-Poisson equations to incompressible type Euler equations," Journal of Mathematical Analysis and Applications, vol. 342, no. 2, pp. 1107-1125, 2008.

[11] Y. Brenier, "Convergence of the Vlasov-Poisson system to the incompressible Euler equations," Communications in Partial Differential Equations, vol. 25, no. 3-4, pp. 737-754, 2000.

[12] S. Wang and S. Jiang, "The convergence of the Navier-Stokes-Poisson system to the incompressible Euler equations," Communications in Partial Differential Equations, vol. 31, no. 4-6, pp. 571-591, 2006.

[13] A. Jüngel and S. Wang, "Convergence of nonlinear Schrödinger-Poisson systems to the compressible Euler equations," Communications in Partial Differential Equations, vol. 28, no. 5-6, pp. 1005-1022, 2003.

[14] H. Brézis, F. Golse, and R. Sentis, "Analyse asymptotique de l'équation de Poisson couplée à la relation de Boltzmann. Quasi-neutralité des plasmas," Comptes Rendus de l'Académie des Sciences. Série I. Mathématique, vol. 321, no. 7, pp. 953-959, 1995. 
[15] L. Hsiao, P. A. Markowich, and S. Wang, "The asymptotic behavior of globally smooth solutions of the multidimensional isentropic hydrodynamic model for semiconductors," Journal of Differential Equations, vol. 192, no. 1, pp. 111-133, 2003.

[16] P. Crispel, P. Degond, and M.-H. Vignal, "An asymptotic preserving scheme for the two-fluid EulerPoisson model in the quasineutral limit," Journal of Computational Physics, vol. 223, no. 1, pp. 208-234, 2007.

[17] G. Q. Chen, J. W. Jerome, and D. H. Wang, “Compressible Euler-Maxwell equations,” Transport Theory and Statistical Physics, vol. 29, pp. 311-331, 2000.

[18] J. W. Jerome, "The Cauchy problem for compressible hydrodynamic-Maxwell systems: a local theory for smooth solutions," Differential and Integral Equations, vol. 16, no. 11, pp. 1345-1368, 2003.

[19] Y. J. Peng and S. Wang, "Convergence of compressible Euler-Maxwell equations to compressible Euler-Poisson equations," Chinese Annals of Mathematics, Series B, vol. 28, no. 5, pp. 583-602, 2007.

[20] T. Kato, "Nonstationary flows of viscous and ideal fluids in $\mathbb{R}^{3}$," Journal of Functional Analysis, vol. 9, no. 3, pp. 296-305, 1972.

[21] S. Klainerman and A. Majda, "Singular limits of quasilinear hyperbolic systems with large parameters and the incompressible limit of compressible fluids," Communications on Pure and Applied Mathematics, vol. 34, no. 4, pp. 481-524, 1981.

[22] S. Klainerman and A. Majda, "Compressible and incompressible fluids," Communications on Pure and Applied Mathematics, vol. 35, no. 5, pp. 629-651, 1982. 


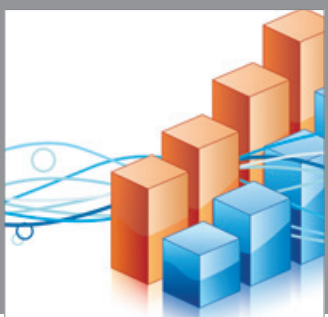

Advances in

Operations Research

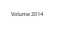

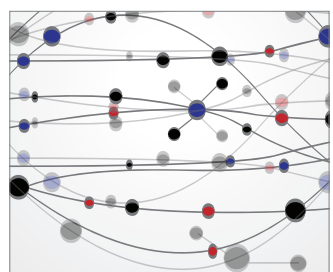

\section{The Scientific} World Journal
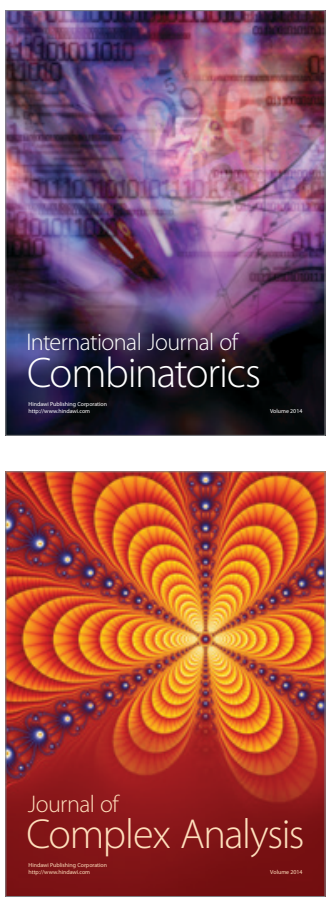

International Journal of

Mathematics and

Mathematical

Sciences
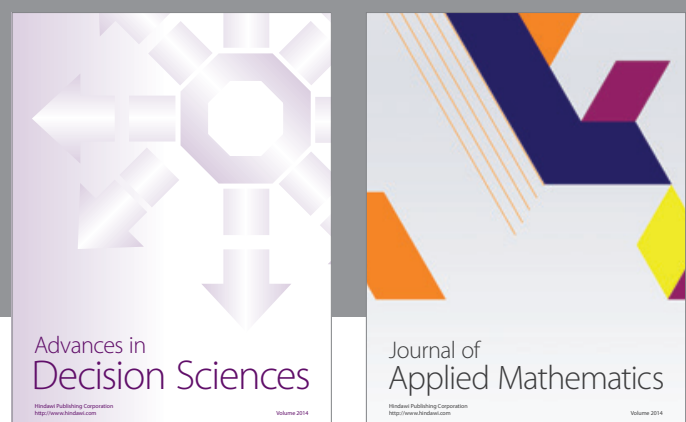

Journal of

Applied Mathematics
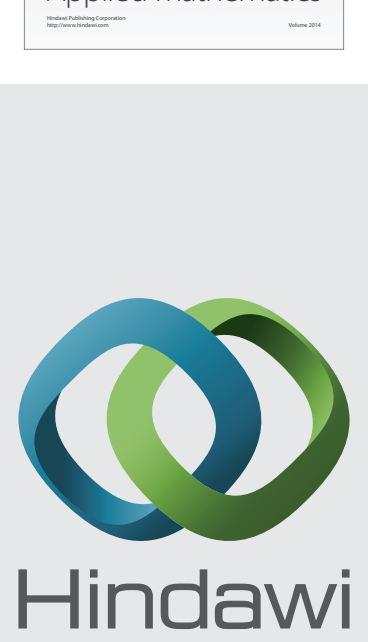

Submit your manuscripts at http://www.hindawi.com
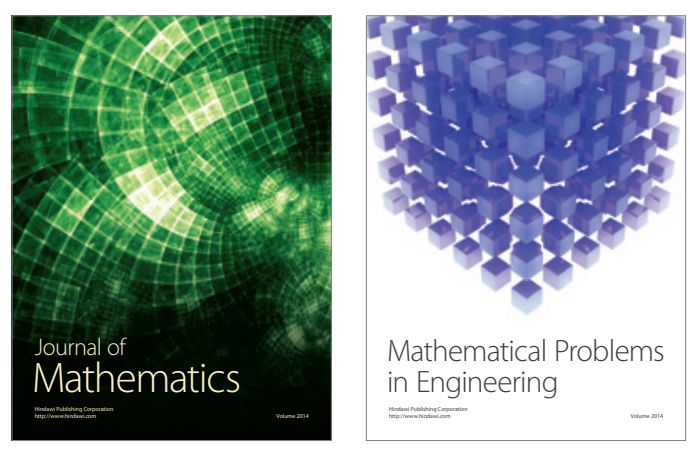

Mathematical Problems in Engineering
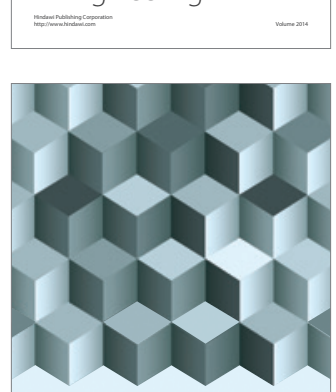

Journal of

Function Spaces
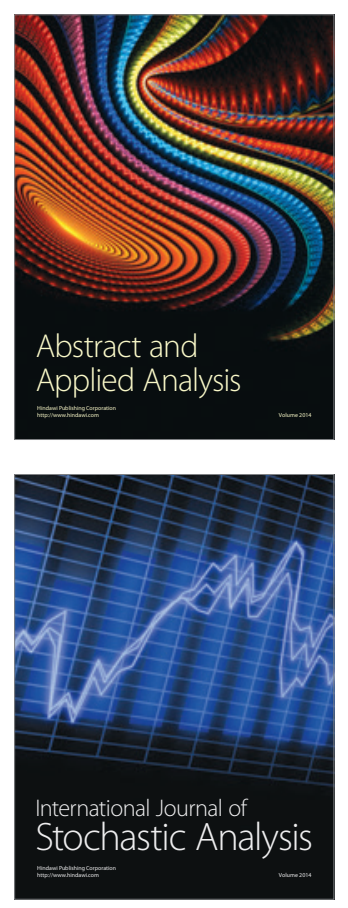

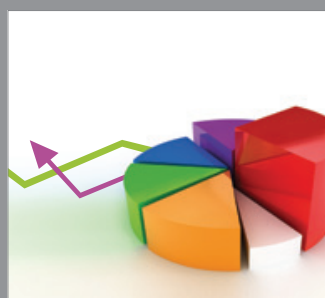

ournal of

Probability and Statistics

Promensencen
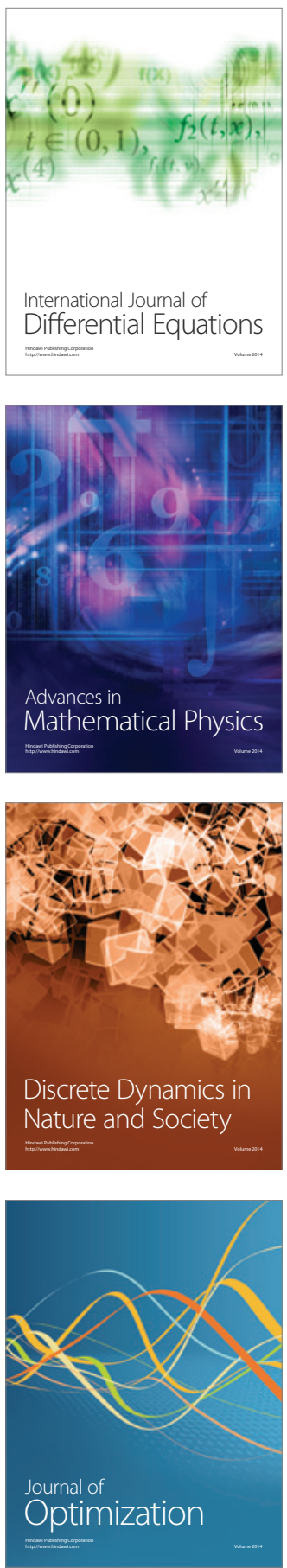